\title{
Dynamic Left Ventricular Outflow Tract Obstruction in Dogs Responding to $\beta 1$-Adrenergic Antagonist Treatment
}

Hyung-Kyu Chae ( $\nabla$ vetchae@snu.ac.kr)

Laboratory of Veterinary Internal Medicine, College of Veterinary Medicine, Seoul National University,

Seoul https://orcid.org/0000-0002-6876-9460

Won-Kyoung Yoon

Guardian Angel Veterinary Hospital, Seoul

Seunggon Lee

Seoul Animal Heart Hospital, Seoul

Hwa-Young Youn

Laboratory of Veterinary Internal Medicine, College of Veterinary Medicine, Seoul National University,

Seoul

\section{Short Report}

Keywords: Canine, Cardiac hypertrophy, Obstruction, $\beta$-blockers

Posted Date: February 18th, 2022

DOI: https://doi.org/10.21203/rs.3.rs-1360486/v1

License: (c) (1) This work is licensed under a Creative Commons Attribution 4.0 International License.

Read Full License 


\section{Abstract}

Dynamic left ventricular outflow tract obstruction (DLVOTO) is commonly observed in feline patients with hypertrophic cardiomyopathy, but it is less frequent in dogs. DLVOTO observed in young dogs often resolves after $\beta 1$-adrenergic antagonist treatment. However, due to the lack of reported cases and the absence of a control group, it is difficult to determine whether improvements are due to aging-related changes in ventricular architecture or treatment itself. Thus, we report two cases of DLVOTO confirmed via echocardiography. Case 1 was an 8-month-old female Spitz and Case 2 was a 5-month-old female Cocker Spaniel. A prophylactic $\beta 1$-adrenergic antagonist was prescribed to reduce the outflow gradient, and echocardiographic changes associated with DLVOTO and consequent left ventricular concentric hypertrophy resolved after $\beta 1$-blocker prescription. This is the first report to discuss echocardiographic changes in relation to changes in $\beta$-blocker dose in canine DLVOTO.

\section{Introduction}

Dynamic left ventricular outflow tract obstruction (DLVOTO) is commonly observed in cats with hypertrophic cardiomyopathy (HCM) (Sisson 1992; Fox et al. 1995). Given the low incidence of HCM in dogs, DLVOTO primarily occurs secondary to congenital aortic stenosis or mitral valve (MV) insufficiency (Sisson 1992; De Majo et al. 2003), although it can be acquired transiently due to hypovolemia-induced decreases in left ventricular volume (Aoki et al. 2015; Paige et al. 2007).

LVOTO of the septal MV leaflet is confirmed on echocardiography based on increases in LVOT velocity. Systolic anterior MV motion can be observed on real-time two-dimensional (2D) images by an experienced cardiologist/radiologist and can be detected more easily on M-mode images of the MV. In the Doppler LVOT spectrum, a late-peaking ventricular outflow profile with a "scimitar" shape may also be observed. Such occlusion can also result in abnormal increases in ventricular septum and left ventricular wall thickness. Although these findings are similar to those of primary $\mathrm{HCM}$, the conditions differ significantly in terms of treatment course and prognosis. LVH in DLVOTO occurs secondary to compensation for obstruction and resolves as the obstruction subsides. However, LVH in HCM is a primary cause of diastolic dysfunction, and DLVOTO is often caused by LVH. Since HCM and LVH induced by DLVOTO lead to fatal or benign outcomes, respectively, a careful diagnostic process is required to avoid misinterpretation of the findings.

In human cases of non-HCM DLVOTO, $\beta$-blockers can be used as first-line therapy (Al-Nasser et al. 2002; Gregor and Čurila 2015). However, there are few reports of DLVOTO in dogs, and a detailed classification of its causes and appropriate treatment protocol are lacking. DLVOTO in dogs with aortic stenosis can result in death or euthanasia after diagnosis (Palacio et al. 1998), while the occurrence and resolution of DLVOTO in dogs without aortic stenosis has been rarely reported (Connolly and Boswood 2003; Loureiro et al. 2008). All nine dogs in these previous papers were under 1 year old, and four were terrier breeds. DLVOTO resolved after $\beta$-blocker treatment or as the dogs aged without treatment (Connolly and Boswood 2003; Loureiro et al. 2008). However, a clear breed predisposition, the precise effects of $\beta$ - 
blocker, and standard treatment regimens for DLVOTO in dogs without subvalvular aortic stenosis (SAS) have not been established given the paucity of reported cases. Further, the studies did not include control groups, making it difficult to confirm whether the outflow obstruction resolves due to $\beta$-blocker treatment or age-related changes in ventricular structure. Thus, we report canine cases of DLVOTO where we observed a response based on prescribed $\beta$-blockers.

\section{Case History}

\section{Case 1}

An 8-month-old female Spitz was referred for evaluation of the cause of a heart murmur identified during routine physical examination. The owner indicated that the dog showed no clinical symptoms related to heart murmur. On physical examination, a grade IV/VI systolic murmur with a point of maximal intensity (PMI) located near the heart base was auscultated. Mucous membrane colour and peripheral pulse quality were normal, and no pulse deficits were noted. The dog's systolic blood pressure was $110 \mathrm{mmHg}$, which was measured using a non-invasive Doppler measuring device (Doppler Flow Detector, Model 811B, Parks Medical, Aloha, OR) with a cuff placed at the tail. There were no evident abnormalities in the blood analysis.

Thoracic radiography revealed a normal heart size (vertebral heart score [VHS]: 9.8v) and a normal pulmonary parenchyma. LVH and systolic anterior motion of the MV were observed in the right parasternal long-axis view of the 2D echocardiography mode. The MV apparatus appeared normal, and no subaortic/aortic stenosis was observed on any $2 \mathrm{D}$ echocardiographic views. When measuring the left ventricle during systolic and diastolic assessment in M-mode and 2D mode of the right parasternal shortaxis view, end-diastolic septum thickness (IVSd) and left ventricular end-diastolic posterior wall thickness (LVPWd) were increased to $7.66 \mathrm{~mm}$ and $9.93 \mathrm{~mm}$, respectively. When the blood flow velocity was evaluated using the Doppler device in the left long-axis view, the MV E peak velocity was $0.66 \mathrm{~m} / \mathrm{s}$, and the Vmax in the LVOT was $5.2 \mathrm{~m} / \mathrm{s}$. Blood flow in the LVOT represented by continuous Doppler exhibited a classic scimitar-shape. Diastolic dysfunction was predicted based on the inverted $E / A$ and $E^{\prime} / A^{\prime}$ ratios (Fig. 1a-e). All echocardiographic examinations (GE Vivid 7 Dimension, GE Healthcare, Chicago, IL) were performed by the same trained veterinarian, and the dog was in a non-excited state, requiring only light physical restraint. Although the cause of DLVOTO and LVH was unclear, we suspected a form of congenital anomaly. $\beta$-adrenergic antagonist (atenolol, $0.5 \mathrm{mg} / \mathrm{kg}$ PO per $12 \mathrm{~h}$ ) was prophylactically administered to reduce the outflow gradient. After 2 months, the dose was increased (atenolol $1 \mathrm{mg} / \mathrm{kg}$ PO per $12 \mathrm{~h}$ ).

After 2 months at the new dose, thoracic radiography revealed a normal heart size (VHS: 10.2v), and echocardiography revealed significant decreases in LVOT obstruction and LV wall thickness. The murmur was no longer auscultated throughout the heart. The Vmax in the LVOT was reduced to $1.3 \mathrm{~m} / \mathrm{s}$, and IVSd and LVPWd decreased from $7.66 \mathrm{~mm}$ to $5.11 \mathrm{~mm}$ and from $9.33 \mathrm{~mm}$ to $7.38 \mathrm{~mm}$, respectively (Table 1). 
Table 1

Changes in echocardiographic parameters according to the course of treatment in Case 1

\begin{tabular}{|c|c|c|c|c|c|}
\hline & Day 0 & Day 113 & Day 195 & Day 293 & Day 384 \\
\hline $\begin{array}{l}\text { LVOT Vmax } \\
\text { velocity }\end{array}$ & $\begin{array}{l}5.20 \\
\mathrm{~m} / \mathrm{s}\end{array}$ & $1.32 \mathrm{~m} / \mathrm{s}$ & $5.86 \mathrm{~m} / \mathrm{s}$ & $1.23 \mathrm{~m} / \mathrm{s}$ & $1.22 \mathrm{~m} / \mathrm{s}$ \\
\hline IVSd & $\begin{array}{l}7.66 \\
\mathrm{~mm}\end{array}$ & $5.11 \mathrm{~mm}$ & $8.23 \mathrm{~mm}$ & $6.52 \mathrm{~mm}$ & $4.82 \mathrm{~mm}$ \\
\hline LVPWd & $\begin{array}{l}9.93 \\
\mathrm{~mm}\end{array}$ & $7.38 \mathrm{~mm}$ & $10.21 \mathrm{~mm}$ & $8.51 \mathrm{~mm}$ & $7.94 \mathrm{~mm}$ \\
\hline Atenolol dose & - & $\begin{array}{l}1 \mathrm{mg} / \mathrm{kg} \text { per } \\
12 \mathrm{~h}\end{array}$ & $\begin{array}{l}0.5 \mathrm{mg} / \mathrm{kg} \text { per } \\
12 \mathrm{~h}\end{array}$ & $\begin{array}{l}1 \mathrm{mg} / \mathrm{kg} \text { per } \\
12 \mathrm{~h}\end{array}$ & $\begin{array}{l}1 \mathrm{mg} / \mathrm{kg} \text { per } \\
12 \mathrm{~h}\end{array}$ \\
\hline Septal E'/A' & 0.578 & & & & 0.715 \\
\hline Mitral E/A & 0.85 & & & & 1.19 \\
\hline
\end{tabular}

Based on this dramatic improvement in the obstruction and LV wall thickness on echocardiography, the dose of $\beta$-adrenergic antagonist was reduced (atenolol $0.5 \mathrm{mg} / \mathrm{kg}$ PO per $12 \mathrm{~h}$ ) to ensure use of the minimum medication required.

Three months after reducing the dose, the systolic murmur was again auscultated with a grade of III/VI and a PMI located near the left heart base. Although a normal-sized heart (VHS: 9.7v) was observed on thoracic radiography, echocardiography showed LVOT obstruction and worsening of LV wall thickening. The Vmax of LVOT increased again to $5.86 \mathrm{~m} / \mathrm{s}$. The degree of LVH, which is expected to have occurred secondary to DLVOTO, also increased again. IVSd and LVPWd increased to $8.23 \mathrm{~mm}$ and $10.21 \mathrm{~mm}$, respectively. Considering these findings, the dose of $\beta$-adrenergic antagonist was re-increased (atenolol 1 $\mathrm{mg} / \mathrm{kg}$ PO per $12 \mathrm{~h}$ ). After another 3-6 months, the LVOT obstruction had resolved, and Vmax had dramatically improved to $1.23-1.34 \mathrm{~m} / \mathrm{s}$. As the LVOT obstruction resolved, LV wall thickening also improved. LVOT velocity and end-diastolic left ventricular wall thickness were decreased by $77 \%$ and $20 \%$, respectively, compared with day 0 . The septal E'/A' and mitral E/A ratios in the Doppler study were 0.715 and 1.19, respectively (Fig. 2a-f, Table 1). Since then, the dog has been re-evaluated every 3-6 months and has been taking the same dose of drugs. At the last follow-up, a normal laminar flow shape and blood flow rate through the LVOT were observed during regular echocardiography.

\section{Case 2}

A 5-month-old female Cocker Spaniel was referred to a veterinary cardiologist for evaluation of the heart disease. During physical examination, a grade IV/VI systolic murmur was auscultated on the left side, and respiratory abnormalities were reported by the owner. There were no other physical examination 
findings, and the systolic blood pressure was $140 \mathrm{mmHg}$ when measured with a cuff on the left hind leg. Chest radiography, echocardiography, and blood analysis were performed to determine whether respiratory abnormalities were related to heart murmur. On echocardiography, LVOT was predicted to be 3-4 m/s and severe systolic anterior motion (SAM) was observed (Fig. 3a). The size of LA was normal, but severe LV hypertrophy was observed. Lungs with an interstitial alveolar pattern were observed on chest radiograph. There were no obvious abnormalities in blood analysis. Based on these test results, pneumonia was considered as the cause of respiratory symptoms, and DLVOTO and the resulting abnormal flow into the LVOT were expected to be the cause of cardiac murmur. A $\beta$-adrenergic antagonist (atenolol, $0.5 \mathrm{mg} / \mathrm{kg}$ per PO per $12 \mathrm{~h}$ ) and broad-spectrum antibiotics (enrofloxacin, $2.5 \mathrm{mg} / \mathrm{kg}$ PO per 12 h) were prescribed. After 2 weeks, the murmur was no longer auscultated, and the symptoms of respiratory abnormalities were significantly improved. The interstitial alveolar pattern seen on chest radiograph was reduced, and the peak velocity of LVOT also decreased from 3-4 m/s to around $1 \mathrm{~m} / \mathrm{s}$ (Fig. 3b). All echocardiographic examinations (GE Vivid 7 Dimension, GE Healthcare) were performed by the trained veterinarian cardiologists, and the dog was in a non-excited state, requiring only light physical restraint.

\section{Discussion}

In these cases, congenital DLVOTO was thought to be the main cause of murmur and LVH. This is because (1) there was no dilation of the left atrium or systolic dysfunction, which characterise pathological HCM. Furthermore, (2) the SAM of the MV, LVH, murmur and diastolic dysfunction through a Doppler study improved after $\beta$-blocker treatment. Through continuous echocardiographic analysis according to the drug dose, it was possible to predict that LVH was not due to primary myocardial disorder, but that it was secondary to DLVOTO.

$\beta$-blockers are known to stabilize cardiac function by reducing the output of the sinus node and heart rate. Moreover, $\beta$-blockers may ameliorate the outflow gradient, and the association between $\beta$-blocker treatment and clinical improvement in human cases of non-HCM DLVOTO has been well documented (AlNasser et al. 2002; Ranasinghe et al. 2011). However, in veterinary medicine, an appropriate treatment protocol for DLVOTO has not been established. This is thought to be because there are few reported cases and it is difficult to clearly objectify the benefits of treatment, unlike improvement of chest pain in humans.

As in these cases, variable-type obstructive hypertrophic cardiomyopathy can respond to $\beta$-blocker by reducing myocardial stress and heart rate. Although it was not possible to objectively determine whether clinical symptoms subsided as DLVOTO resolved, our findings suggest that echocardiographic confirmation of improved blood flow and LVH following $\beta$-blocker treatment can be used to evaluate the treatment effect in dogs. In Case 1, DLVOTO status improved after increasing the dose, while certain doses were associated with lack of improvement. In accordance with human findings, this suggests that increasing the $\beta$-blocker dose or adding other negative ionotropic agents can improve DLVOTO in dogs. In 
Case 2, improvement of symptoms and blood flow could be derived due to an appropriate approach to the causes of respiratory abnormalities and heart murmurs (broad-spectrum antibiotics, $\beta$-antagonist)

This case report has limitations. Although congenital SAS was excluded through echocardiography, it was difficult to determine the exact cause of DLVOTO, and post-mortem autopsy was not possible. Further, improvements in DLVOTO were confirmed using continuous echocardiographic data according to the prescribed $\beta$-blocker dose, but studies on their association with clinical symptoms and benefits are lacking.

Nonetheless, our findings indicate that increasing the dosage of $\beta$-blockers may be necessary to attain improvements in DLVOTO in dogs. As in the treatment of DLVOTO due to non-HCM in humans, these dogs experienced positive changes with improved blood flow because of the administration of $\beta$-blockers. These cases will help clinicians not to make an erroneous prognosis as a fatal form of cardiomyopathy in dogs with mild respiratory symptoms and murmur. Further studies are required to determine the association between DLVOTO improvement and clinical symptoms, and to identify the long-term benefits of treating DLVOTO using $\beta$-blockers.

\section{Conclusion}

In both cases, improved blood flow in response to $\beta$-blockers prescription and dose escalation was confirmed by well-trained veterinary cardiologists. This study will be helpful as a reference for treatment approaches in cases of variable-type obstructive myocardial disease in dogs.

\section{Declarations}

\section{Acknowledgements}

This study was supported by the Research Institute for Veterinary Science and BK21 PLUS Program for Creative Veterinary Science Research.

\section{Funding}

The authors declare that no funds, grants, or other support were received during the preparation of this manuscript.

\section{Competing Interests}

The authors have no relevant financial or non-financial interests to disclose.

\section{Author Contributions}

All authors contributed to the study design. The first draft of the manuscript was written by Hyung-Kyu Chae and all authors commented on previous versions of the manuscript. Data collection and analysis 
were performed by Hyung-Kyu Chae, Won-Kyoung Yoon, and Seunggon Lee. Echocardiography was performed by experienced veterinary cardiologists Won-Kyoung Yoon and Seunggon Lee. Supervision and advice on the original draft were provided by Won-Kyoung Yoon and Hwa-Young Youn.

\section{Data Availability}

The datasets generated during and/or analysed during the current study are available from the corresponding author on reasonable request.

\section{Ethics Approval}

This study did not require official or institutional ethical approval.

\section{Consent to participate}

All authors participated voluntarily in this study.

\section{Consent to Publish}

All authors read and approved the final manuscript.

\section{References}

1. Al-Nasser F, Duncan A, Sharma R, O'Sullivan C, Coats AJ, Anker SD, Henein, MY (2002) Beta-blocker therapy for dynamic left-ventricular outflow tract obstruction. Int J Cardiol 86:199-205. https://doi.org/10.1016/s0167-5273(02)00312-1

2. Andersson C, Shilane D, Go AS, Chang TI, Kazi D, Solomon MD, Boothroyd DB, Hlatky MA (2014) Beta-blocker therapy and cardiac events among patients with newly diagnosed coronary heart disease. J Am Coll Cardiol 64:247-252. https://doi.org/10.1016/j.jacc.2014.04.042

3. Aoki T, Sunahara H, Sugimoto K, Ito T, Kanai E, Neo S, Fujii Y, Wakao Y (2015) Dynamic left ventricular outflow tract obstruction secondary to hypovolemia in a German Shepard dog with splenic hemangiosarcoma. J Vet Med Sci 77:1187-1190. https://doi.org/10.1292/jvms.14-0508

4. Connolly DJ, Boswood A (2003) Dynamic obstruction of the left ventricular outflow tract in four young dogs. J Small Anim Pract 44:319-325. https://doi.org/10.1111/j.1478-5827.2003.tb00162.x

5. De Majo M, Britti D, Masucci M, Niutta PP, Pantano V (2003) Hypertrophic obstructive cardiomiopathy associated to mitral valve dysplasia in the Dalmatian dog: two cases. Vet Res Commun 27:391-393. https://doi.org/10.123/b:verc.0000014187.46101.01

6. del Palacio MJF, Bayón A, Bernal LJ, Cerón JJ, Navarro JA (1998) Clinical and pathological findings of severe subvalvular aortic stenosis and mitral dysplasia in a rottweiler puppy. J Small Anim Pract 39:481-485. https://doi.org/10.1111/j.1478-5827.1998.tb03683.x

7. Fox PR, Liu SK, Maron BJ (1995) Echocardiographic assessment of spontaneously occurring feline hypertrophic cardiomyopathy: an animal model of human disease. Circulation 92:2645-2651. 
https://doi.org/10.1161/01.cir.92.9.2645

8. Gregor P, Čurila K (2015) Medical treatment of hypertrophic cardiomyopathy - What do we know about it today? Cor Vasa 57:e219-e224. https://doi.org/10.1016/j.crvasa.2015.02.003

9. Loureiro J, Smith S, Fonfara S, Swift S, James R, Dukes-McEwan J (2008) Canine dynamic left ventricular outflow tract obstruction: assessment of myocardial function and clinical outcome. J Small Anim Pract 49:578-586. https://doi.org/10.1111/j.1748-5827.2008.00623.x

10. Paige CF, Abbott JA, Pyle RL (2007) Systolic anterior motion of the mitral valve associated with right ventricular systolic hypertension in 9 dogs. J Vet Cardiol 9:9-14. https://doi.org/j.jvc.2006.08.003

11. Ranasinghe I, Yeoh T, Yiannikas J (2011) Negative ionotropic agents for the treatment of left ventricular outflow tract obstruction due to sigmoid septum and concentric left ventricular hypertrophy. Heart Lung Circ 20:579-586. https://doi.org/10.1016/h.hlc.2011.05.002

12. Sisson D (1992) Fixed and dynamic subvalvular aortic stenosis in dogs. In: Bonagura JD (ed) Kirk's Current Veterinary Therapy XIII: Small Animal Practice. Saunders, Philadelphia.

\section{Figures}



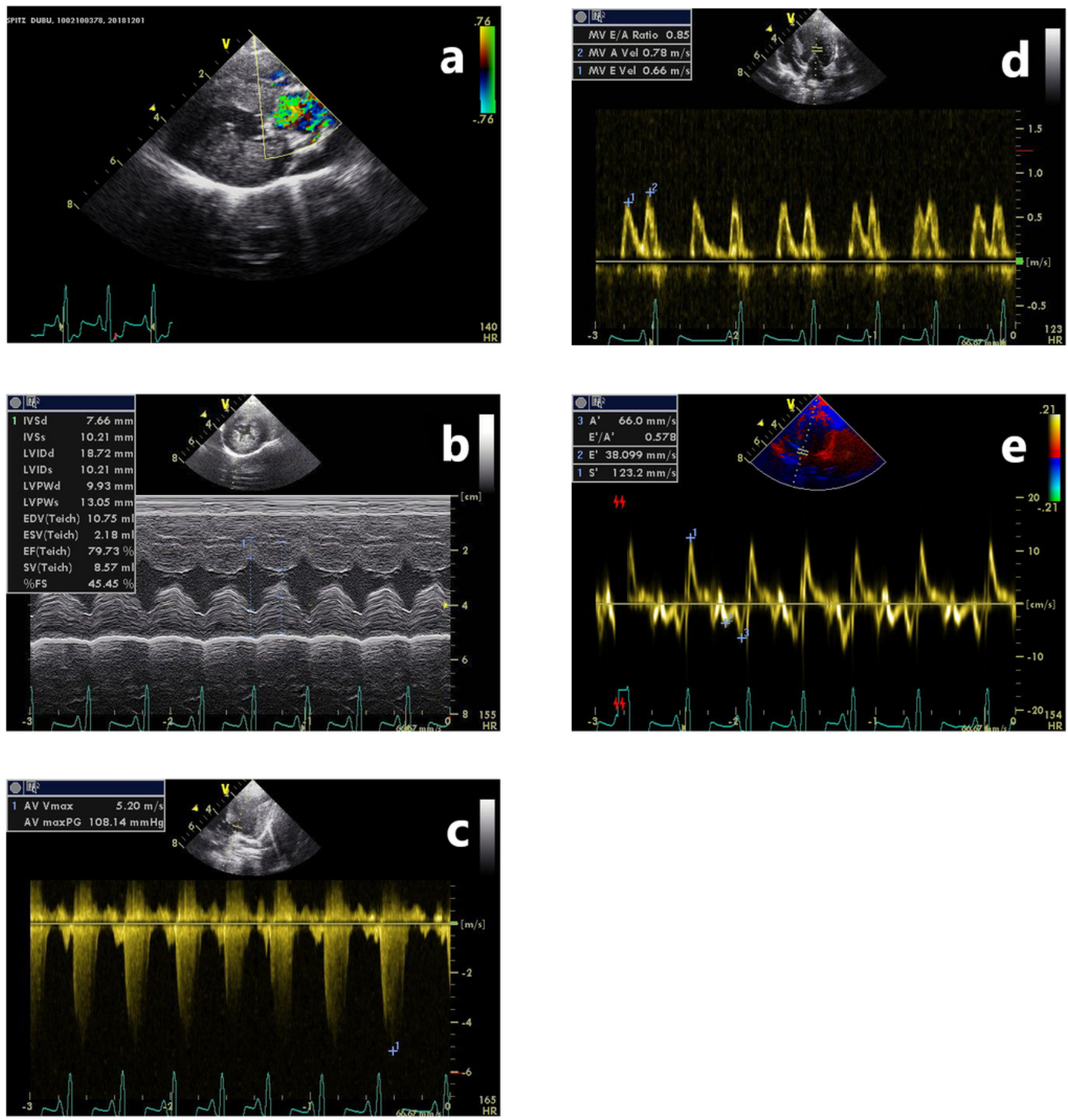

Figure 1

Echocardiography for Case 1 at the first visit. (a) Systolic anterior motion of the mitral valve and consequent occlusion of the left ventricular outflow tract were observed. (b) Left ventricular wall hyperplasia was observed in two-dimensional and M-mode echocardiography. (c-d) When analysing the blood flow in the left ventricular outflow tract through a Doppler test, we observed a late peak with a 
maximum velocity of $5.2 \mathrm{~m} / \mathrm{s}$. (e) Septal tissue Doppler imaging showing E'/A' reversal and normal S' velocity.
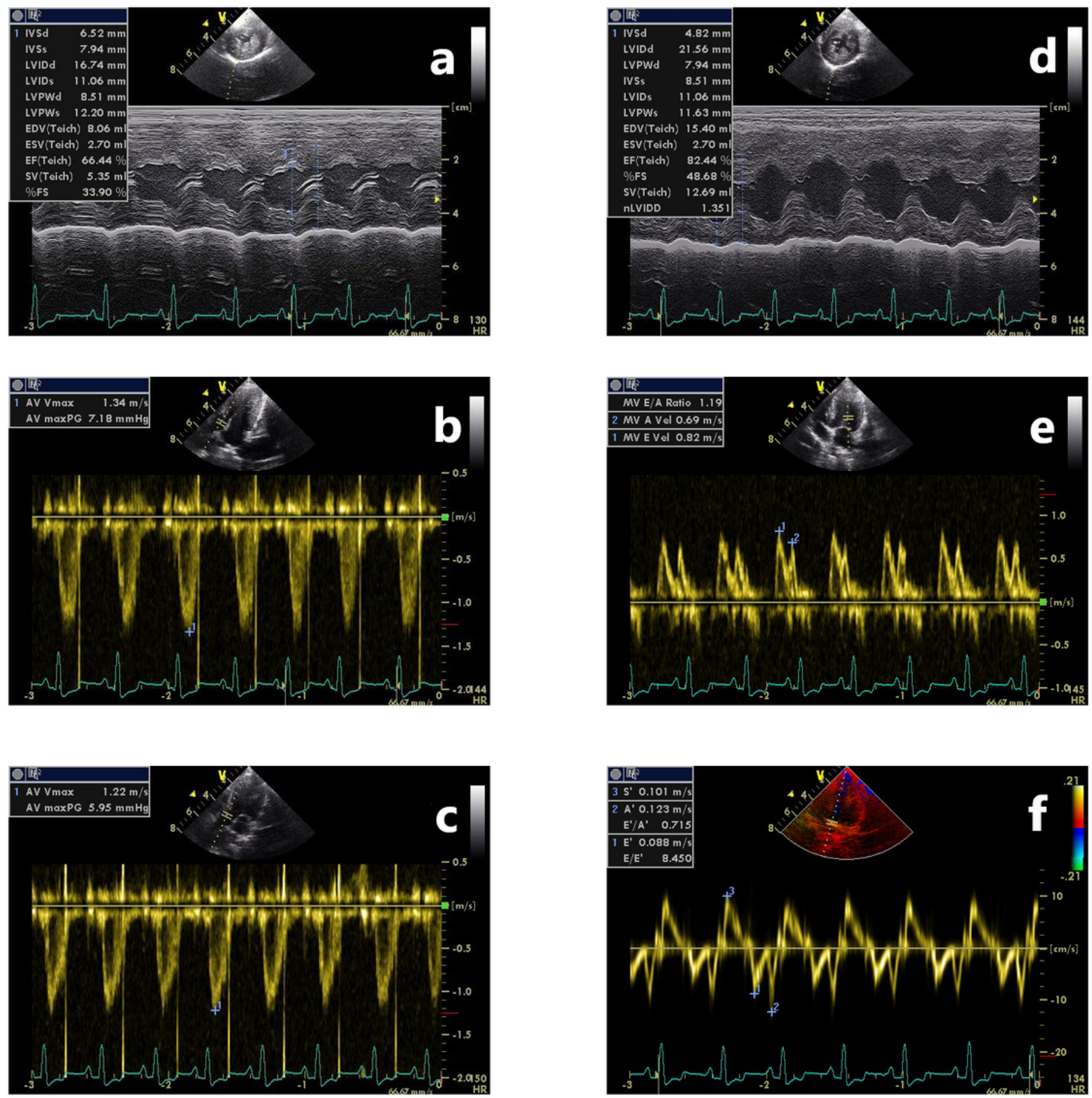

Figure 2

Upon reassessment 3-6 months after re-escalation of the $\beta 1$-adrenergic antagonist dose in Case 1, (a) blockage of the left ventricular outflow tract had resolved, and (b-c) Vmax had again significantly improved to $1.22-1.34 \mathrm{~m} / \mathrm{s}$. (b-c) Normal laminar shapes of the left ventricular outflow tract were 
observed on echocardiography. (d) The degree of left ventricular wall hyperplasia had decreased again. (e-f) The septal E'/A' and mitral E/A ratios increased compared to day 0.
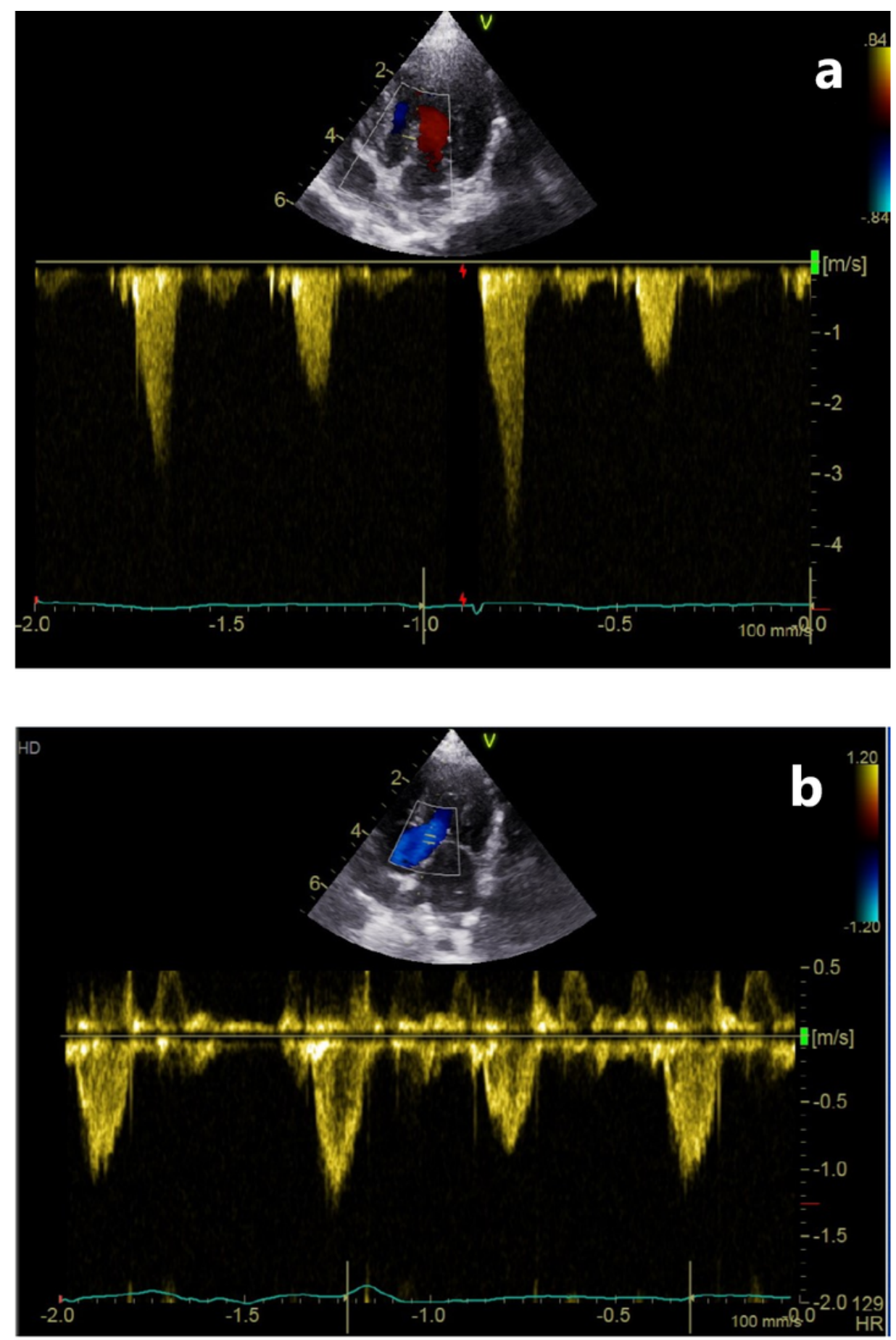

Figure 3

Case 2 Echocardiography in Case 2 before (a) and after (b) $\beta 1$-adrenergic antagonist prescription. Reductions in peak velocity in the LVOT left ventricular outflow tract were observed. 
Page 12/12 